\title{
PATIENT'S KNOWLEDGE IN RHEUMATOID ARTHRITIS: A STUDY OF ASSOCIATION WITH DISEASE ACTIVITY AND FUNCTIONALITY
}

\author{
Naiana Diaz ${ }^{1, \star}$, Eduarda Frare ${ }^{1}$, Barbara Stadler Kahlow ${ }^{1}$ Thelma Larocca Skare ${ }^{1}$, Laís Zanlorenzi ${ }^{1}$ \\ 1.Hospital Universitário Evangélico Mackenzie, Curitiba (PR), Brazil. \\ *Corresponding author: naiana.diaz@gmail.com
}

\section{BACKGROUND}

Rheumatoid arthritis (RA) is an autoimmune disease characterized by the involvement of the synovial membrane mainly of peripheral joints. It is relevant to evaluate whether the patient's degree of knowledge about RA interferes with the function and control of the disease, and patient's quality of life. The aim of this study was to evaluate whether the patient's degree of knowledge about RA interferes with patient function, disease control and quality of life.

\section{METHODS}

Observational cross-sectional study of patients with an established diagnosis of RA. Epidemiological data were collected, disease activity (measured by DAS-28 ESR and DAS-28 CRP), functionality (measured by health assessment questionnaire [HAQ]) and quality of life (measured by short form health survey with 12 questions [SF-12]). To evaluate the knowledge of the disease, the patient's knowledge questionnaire (PKQ) was applied. The PKQ has four domains: 1 ) about etiology and laboratorial tests (rated from 0 to 10); 2) about medications used for treatment (rated from 0 to 6); 3) about exercising (rated from 0 to 6); 4) about joint protection and energy conservation (rated from 0 to 8 ). The global score ranges from 0 (worst result) to 30 (best result).

\section{RESULTS}

About 101 patients were included (91\% females; median age of 56 years and with median years of formal education of 8 years). In general, the sample had very low knowledge about the disease with median PKQ of 14. The median values for each domain were: four for etiology/laboratorial exams; two for drug treatment; four for exercises; three for joint protection and energy conservation. It was not possible to demonstrate correlation between the level of patient's knowledge and disease activity (DAS-28 VHS, DAS-28 $\mathrm{PCR}$ ), with HAQ or SF-12 (all with $p>0.5$ ). However, a positive correlation was found with the level of education and the total value of PKQ ( $p=0.0005$; rho $=0.33 ; 95 \% \mathrm{Cl}=0.14-0.50)$.

\section{CONCLUSION}

This study demonstrated lack of knowledge about RA and its treatment by the patients. There was no correlation between the level of knowledge of the disease and variables of disease activity or functionality. The number of years of formal study positively influenced the disease knowledge.

\section{KEYWORDS}

Rheumatoid arthritis, Quality of life, Educational management. 